\title{
Signal Transduction Mechanisms Underlying Growth Hormone Receptor Action
}

\author{
Adrian C. Herington ${ }^{* 1}$ and Peter E. Lobie ${ }^{2}$ \\ ${ }^{I}$ Institute of Health and Biomedical Innovation and Faculty of Health, Queensland University of Technology, Brisbane, \\ QLD, Australia \\ ${ }^{2}$ Cancer Science Institute of Singapore, National University of Singapore Centre for Life Sciences, Singapore
}

\begin{abstract}
Our understanding of the mechanisms of action of GH and its receptor, the GHR, has advanced significantly in the last decade and has provided some important surprises. It is now clear that the GH-GHR axis activates a number of inter-related signalling pathways, not all of which are dependent on the intracellular tyrosine kinase, JAK2 as originally postulated. JAK2-independent pathways, mediated via the Src family kinases, together with a number of negative regulators of GH signalling and emerging cross-talk mechanisms with other growth factor receptors, provide a complex array of mechanisms that are capable of fine-tuning responses to $\mathrm{GH}$ in a cell context dependent manner. Additionally, it is also now clear that GH and the GHR can translocate to the nucleus of target cells and initiate, as yet not well defined, nuclear responses. Continued emphasis on elucidation of these complex mechanisms is critical to provide further insights into the diverse physiological and pathophysiological effects of GH.
\end{abstract}

Keywords: Growth hormone receptor, JAK2, Src kinases, Suppressors of Cytokine Signalling, nuclear translocation.

\section{INTRODUCTION}

The growth hormone receptor (GHR) was first described/detected in tissues by Tsushima \& Friesen in 1973 on the basis of specific high affinity ${ }^{125} \mathrm{I}$-hGH binding studies [1]. Early studies focussed on the functional roles of GHGHR interaction, species specificity of binding, regulation of GHR expression and relationships with various GH-related disorders. With the cloning of the GHR in 1987 by the Genentech team [2], evidence has continued to mount regarding the structure-function relationships, clinically relevant mutations, mechanisms of action, signalling pathways and agonists and antagonists for clinical use.

Over this time a quite remarkable evolution of the functional paradigm has occurred - from GH-induced dimerisation to constitutive homodimerisation, JAK-STAT specific signalling to additional JAK-independent signalling, stand-alone signalling and cross-talk signalling with other growth factor receptors, both endocrine and autocrine activation, and cell membrane versus nuclear localisation and function. Additionally, the identification of the soluble extracellular domain - the GH binding protein, GHBP - and its potential in prolonging the half life of $\mathrm{GH}$ in the circulation and/or influencing its action at the target cell have provided new understandings in the complexities of GH and GHR action.

\footnotetext{
*Address correspondence to this author at the Faculty of Health, Queensland University of Technology, GPO Box 2434, Brisbane QLD, 4001, Australia; Tel: (+617) 3138 2554; Fax: (+617) 3138 6039;

E-mail a.herington@qut.edu.au
}

This review summarises the 2010 state of knowledge regarding the mechanism of action of $\mathrm{GH}$ through its cognate receptor, the GHR.

\section{GHR STRUCTURE AND FUNCTION}

The original view of GH-GHR interactions, based on the crystal structures of GH bound to the extracellular domain of the GHR, was that two GHR molecules were bound by a single GH molecule in a sequential, 2-step binding reaction $[3,4]$. $\mathrm{GH}$, with 2 distinct and asymmetric binding sites on opposite faces of the $\mathrm{GH}$ molecule, bound with differing affinities to a single binding site on each of two GHR molecules. The resultant dimerisation of the GHR which, like other Class 1 cytokine receptors, does not contain an intrinsic kinase domain, then recruited the cytoplasmic kinase, JAK 2, to initiate intracellular signalling [5]. More recent evidence, however, clearly suggests that the GHR exists, in its non-liganded state, as a natural homodimer [6, 7], each GHR molecule already associated with one or more inactive kinases, including JAK2 and Src family kinases, and that binding of $\mathrm{GH}$ occurs in a 2 -step reaction involving rotation and re-orientation of the second GHR molecule [8]. This leads to activation and cross-phosphorylation of the associated kinases, phosphorylation of each GHR cytoplasmic tail and the recruitment and subsequent phosphorylation of cytoplasmic proteins that then trigger further phosphorylation cascades and transcriptional activity (for details see below).

Precise structural and functional knowledge of GH-GHR interaction has led to the development over the past 15 years of a variety of GHR functional agonists and antagonists with 
real or potential application for clinical use [reviewed in 9, 10]. GH modified by covalent attachment of varying amounts of polyethylene glycol (pegylated GH) has led to longer half-lives and longer-acting agonists, although with reduced potency [reviewed in 10]; fusion proteins between $\mathrm{GH}$ and the extracellular domain of the GHR (the GHBP) led to a markedly prolonged half life of the immunoreactive $\mathrm{GH}$ but also, intriguingly, to enhance weight gain and circulating IGF-I in hypophysectomised rats [11]; a variety of mutations within site 1 have demonstrated enhanced receptor potency through increased binding affinity ([12,13]; a G120K mutation in site 2 , coupled with site 1-enhancing mutations and pegylation (B2036-PEG, pegvisomant) has led to a highly potent antagonist that is now in use for treatment of acromegaly [reviewed in 10, 14].

Despite the constitutive dimer structure of the membraneinserted GHR, conformational changes elicited by the 2-site binding of $\mathrm{GH}$ are still required for activation. A GH variant in which the second binding site has been mutated (G120R $\mathrm{GH})$ abrogates binding at this site, acts as a $\mathrm{GH}$ antagonist, and fails to activate GHR signalling $[15,16]$. Detailed structural studies have demonstrated the mechanistic basis for this [9, 17]. Surprisingly, however, a G120R-G120R linked dimer variant that contains two active site 1 domains, but no active site 2 domains, was able to activate GHR signalling in a similar dose and time course relationship to native GH itself, albeit with diminished potency [18]. This suggests that it is not the binding to $\mathrm{GH}$ site 2 per se that triggers the appropriate activating conformational change but that flexibility in the extracellular domain, that allows the cooperative binding of a ligand with two $\mathrm{GH}$ site $1 \mathrm{~s}$, is sufficient for GHR activation.

The GHR contains a number of structural features that define its various activities and characterise it as a distinct member of the class 1 cytokine receptor family [reviewed in 19-21]. The extracellular domain consists of an N-terminal fibronectin-like domain, 3 conserved cysteine pairs and a conserved WSXWS domain (YXXFS in human GHR) in the juxta-membrane extracellular domain which is critical to the conformational change following binding of GH. A single transmembrane domain is followed by two proline-rich regions (so-called Box 1 and Box 2) in the juxta-membrane intracellular domain which are required for both JAK2 association with GHR and subsequent GH-induced JAK2 activation, respectively. Several tyrosine phosphorylation sites in the C-terminal domains represent the activated binding sites for a number of cytoplasmic signalling molecules that result in $\mathrm{GH}$-induced signalling cascades (see below).

JAK2 is now also known to stabilise the GHR dimer, which initially forms in the endoplasmic reticulum, as it translocates through the endoplasmic reticulum and Golgi on its way to the plasma membrane [22] (Fig. 1). JAK2 may also stabilise the membrane location and decrease the constitutive internalisation and down regulation of the GHR [23-25]. In contrast, some studies have disagreed as to whether GH-induced internalisation of the GHR is [26, 27] or is not [28] dependent on JAK activation. The most recent study by Deng et al. [29] suggests that GH-induced GHR downregulation depends on ubiquitination that requires both JAK2 activity and GHR phosphorylation.

A number of variant, alternatively-spliced GHR transcripts have been reported, although the functional implications have not been fully understood. At least 13 hGHR mRNAs are generated through alternative splicing in the 5' untranslated exons, although all splice into a common acceptor site just upstream of the translation start site in exon 2 , thereby each giving rise to a full length receptor. Expression levels vary markedly, some are expressed widely, others in a developmental and/or tissue specific manner [30]. Differential promoter usage leads to variation in transcript expression, with the so-called V2 transcript being the major ubiquitously expressed form. The physiological implications of this developmental and tissuespecific regulation are not clear. An exon-3 deleted form of the GHR has been reported to be widely expressed in human tissues $[31,32]$. This form which lacks amino acids 7-28 at the N-terminus does not affect GHR binding affinity, capacity or internalisation rates, but does influence sensitivity to $\mathrm{GH}$ activation and is associated with enhanced responsiveness to $\mathrm{GH}$ treatment in children [33, 34]. It also predicts an improved response to the antagonist pegvisomant in acromegaly [35].

Naturally occurring, membrane-bound but cytoplasmically-truncated splice variants of the GHR have been reported [36, 37] which, although not having a direct signalling function, may still form natural long-short heterodimers with the full length GHR as demonstrated through transfection studies in 293 cells [37]. The truncated variants inhibited STAT5 signalling of the full length GHR and therefore may play a significant role in modulating the functional activity of the wild-type GHR (Fig. 1). The short form has also been demonstrated to have a greater tendency for proteolytic cleavage of the extracellular domain, thereby providing a ready source of the soluble growth hormone binding protein (GHBP) [37].

\section{THE GROWTH HORMONE BINDING PROTEIN (GHBP)}

The GHBP, produced in primates solely by proteolytic cleavage, as opposed to rodents where an alternatively spliced complementary mRNA transcript is also produced, was first described in rabbit serum [38] but subsequently in human and other species by Baumann et al. [39] and Herington et al. [40]. This was a surprising finding at the time as it was generally thought that unlike the steroid hormones and some peptide hormones eg insulin-like growth factors (IGFs), protein hormones did not require carriage in the circulation by specific binding proteins. Several functions have been ascribed to the GHBP. In addition to carriage in the circulation and increasing the half-life of $\mathrm{GH}$ [41], the GHBP clearly has a role in modulating the delivery of GH to the GHR within the extracellular environment. In this context the soluble, extracellular GHBP could act as a competitive inhibitor and sequester GH away from the GHR. This has been shown to be the case in vitro in both 3T3F442A preadipocytes and in IM-9 lymphocytes [42]. Under 


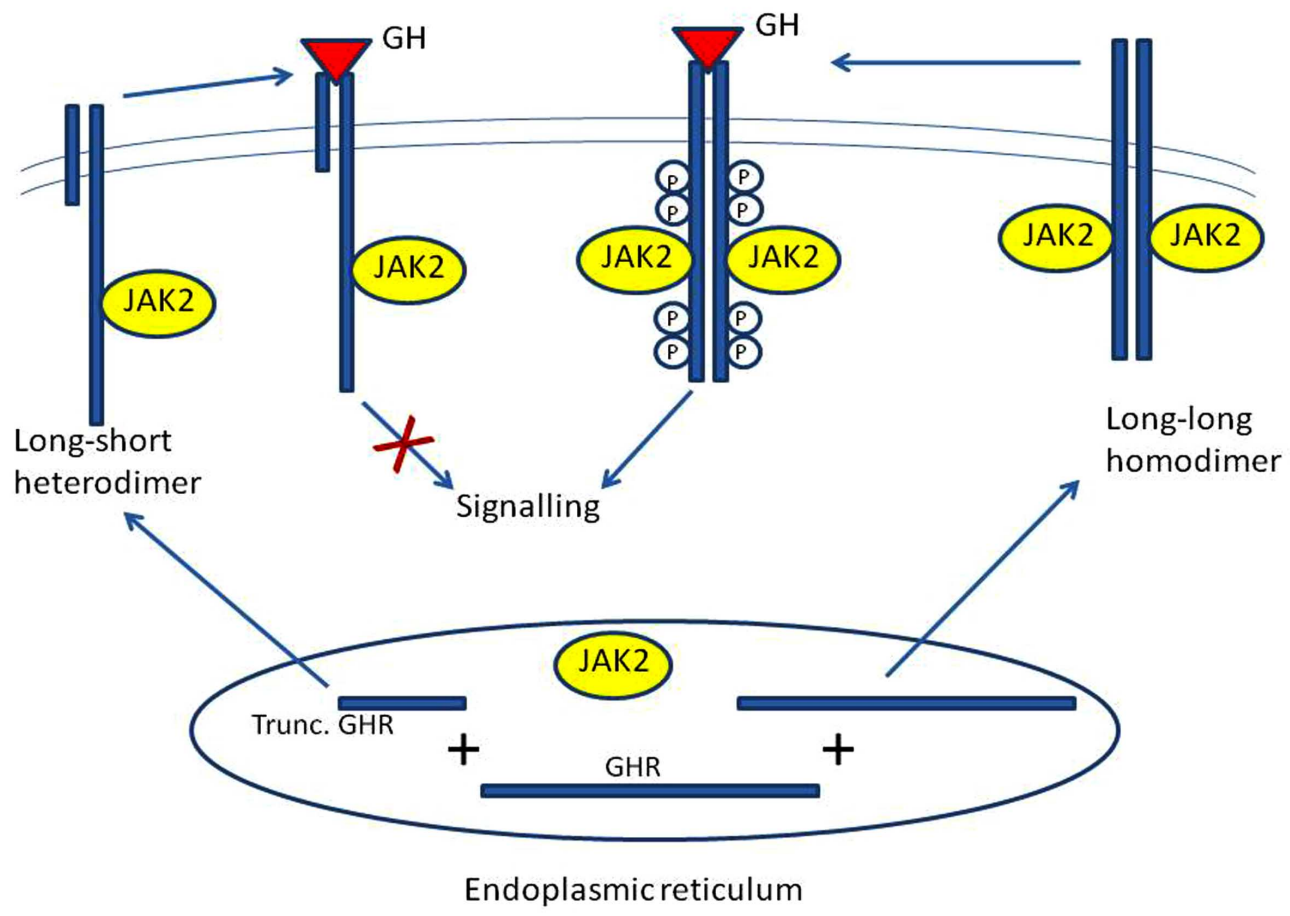

Fig. (1). Schematic of the role of JAK2 in stabilising the initial formation of the GHR dimer in the endoplasmic reticulum, prior to translocation to the plasma membrane. Formation of both the long-long homodimer and the predicted long-short heterodimer variants and their impact on GH-induced signal transduction is illustrated.

the original GH-induced dimer model, it was also suggested that the soluble extracellular GHBP could compete with monomeric membrane-bound forms of the GHR and therefore form (probably) inactive long-short heterodimers, that would fail to signal, or at least signal quite differently from the wild-type long-long homodimers [43]. With the more recent understanding of the intracellular pre-formation of GHR dimers, this model is less tenable, although as mentioned above the existence of the naturally produced membrane-bound truncated splice-variant described by Ross et al. [37] may provide the physiological equivalent of the variant long-short dimers. Overexpression of the truncated form does act as a dominant negative inhibitor of the fulllength GHR [37]. Nonetheless, with the new dimer model paradigm the cellular functional role of the GHBP remains unclear, although suggestions that it may have a role in the nucleus cannot be discarded (see below).

Proteolytic cleavage of the membrane-bound GHR has been demonstrated to be catalysed by ADAM 17 (or TACE) in the proximal membrane region and to release the extracellular domain of the receptor in humans and other species [44]. Cleavage can be induced by a variety of factors, including protein kinase $\mathrm{C}$ activators, some growth factors, serum or endotoxin $[45,46]$ and has been reported to regulate both GHR sensitivity and signalling [22]. The remnant protein (transmembrane plus cytoplasmic domains) intriguingly can be subsequently cleaved by gammasecretase within the transmembrane domain to release the intracellular domain within the cell [47]. This fragment has been detected in both the cell cytosol and nucleus [47], however it is not know if this fragment has intracellular or intranuclear functions via regulation of gene expression (but see below).

\section{GHR SIGNALLING}

Phosphorylation of the several cytoplasmic domain tyrosines following $\mathrm{GH}$ activation leads to activation of several signalling pathways, many of which are common and/or complementary to those of the other recognised Class 1 cytokine receptor mechanisms of action [48]. The major, but not sole, effector of signalling is JAK2 and the main JAK2 pathway involved is that of the Signal Transducers and Activators of Transcription (STATs). Additionally, however, there is considerable evidence now demonstrating both JAK2-independent signalling as well as major inhibitory mechanisms for regulating GH action and GHR signalling through the negative modulators, Suppressors of Cytokine Signalling (SOCS) and Protein Tyrosine Phosphatases (PTPs).

\section{JAK2- Dependent Signalling}

JAK2 is one of four Janus tyrosine kinases [48]. As indicated above, binding of GH to the GHR homodimer activates the kinase domains of both JAK2 molecules bound to the proline-rich Box 1 domains, leading to phosphorylation of each JAK2 at multiple tyrosine residues $[5,49]$. GH-induced phosphorylation of both JAK1 and 3 has also been reported [50,51] although this represents a minor event. Subsequent cross-phosphorylation of tyrosines in the cytoplasmic tails of the two GHR molecules [52] leads to recruitment of a host of other cytoplasmic signalling intermediates harbouring Src homology 2 (SH2)-domains [ 


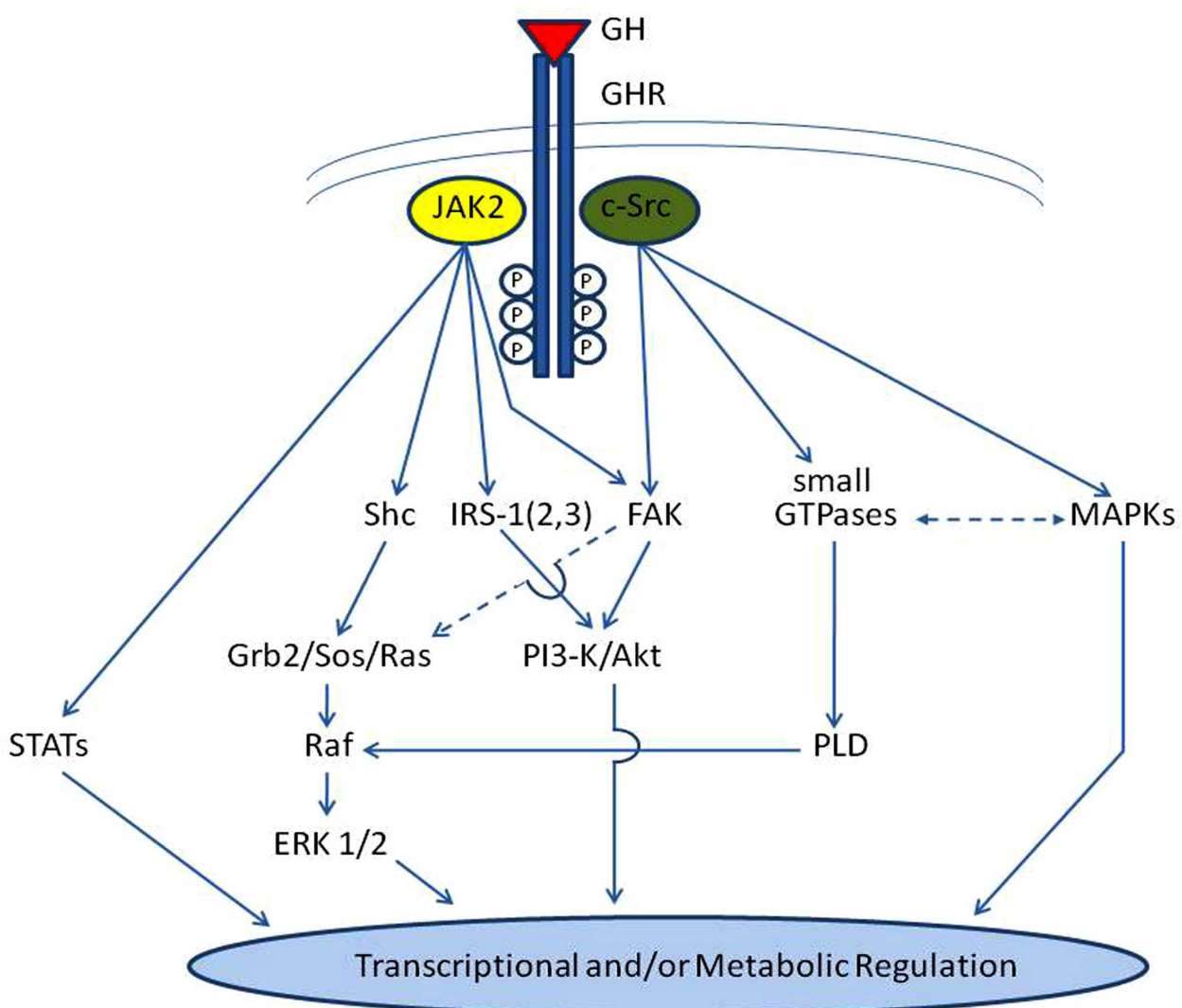

Fig. (2). A simplified model for the JAK2-dependent and Src (JAK2-independent) signalling pathways initiated by GH interaction at the cell membrane. Adapted from [20].

reviewed in detail in 20] (Fig. 2). Recruitment of specific mediators may be dependent on cell type, compartmentalisation and differentiation stage [eg 53]. The transcription factors STAT5 $\mathrm{a}$ and $\mathrm{b}$ are the key mediators of JAK2 activation, although STATs 1 and 3 have also been implicated in some signalling pathways [54].

Following phosphorylation of STAT $5 \mathrm{a}$ and $\mathrm{b}$, they homo- or -heterodimerise, translate to the nucleus and bind directly to STAT responsive elements in promoters of target genes $[54,55]$. The JAK2-STAT 5 pathway has been shown to be central to GH-induced changes in metabolic function, body growth and IGF-I gene transcription [54-56]. Defects in JAK-STAT signalling have been reported to lead to growth retardation in human subjects $[57,58]$.

JAK2 also binds other adaptor proteins independently of the STATs (Fig. 2). Binding of Shc to JAK2 leads to sequential activation of the Grb2-SOS-Ras/Raf- MAPK pathway in which activated ERK1/2 either directly, or via phosphorylation of the transcription factors Elk and/or CEBPbeta, activates specific sets of target genes [59-61].

GH activation also leads to JAK2-dependent phosphorylation of Insulin Receptor Substrate (IRS) proteins 1, 2 and 3, thus activating the PI-3 kinase/Akt pathway [59, 62]. GH also results in Focal Adhesion Kinase (FAK) activation, leading to a $\mathrm{GH}$-induced reorganisation of the actin cytoskeleton in a variety of cell types [63, 64]. FAK activation appears to require association with JAK2 as demonstrated by use of JAK2 inhibitor AG490 [65], although other data suggest the c-Src family of protein kinases may also be essential [66]. FAK also can interact with the PI3-K/Akt pathway [67] and may provide an alternative pathway to IRS stimulated PI3-K/Akt for GH to modulate cell activities in cell type and cell differentiation specific ways.

\section{JAK2-Independent Signalling}

Despite the key role for JAK2 in GHR signalling, evidence continues to mount demonstrating a number of JAK2-independent signalling mechanisms, particularly using Src family kinases. ERK $1 / 2$ activation has been demonstrated to occur through an alternative JAK2independent pathway $[68,69]$. GH-induced c-Src, c-Fyn and Lyn phosphorylation has been shown to activate a number of signalling molecules, including FAK, p130Cas and CrkII all of which are linked to activation of several other key pathways via Grb2 or phospholipase $\mathrm{C}$ gamma and Ras (ERK1/2 pathway), CrkII/Rap1/2 (MAPK/JNK/c-Jun pathway) and the Ras-like small GTPases RalA/RalB (ERK1/2 via phospholipase D) [reviewed in detail in 20] (Fig. 2).

Recent data from Rowlinson et al. [70] have shown that Lyn may be the major Src kinase involved - transfection with Lyn siRNA caused a major decrease in GH-activated ERK. That it was independent of JAK2 was shown in GHR+ve/JAK2-ve $\gamma 2 A$ cells. Human GH-stimulated ERK activation was blocked by the Src inhibitor, PP2. That Lyn 
acts directly via phospholipase $\mathrm{C} \gamma$ was also shown using the PLC inhibitor U73122 [70]. In contrast, GH activation of the small GTPases Rap1/Rap2 can also negatively impact GHstimulated RalA/B and therefore inhibit GH-stimulated Erk $1 / 2$ and Elk-1 mediated transcription. In combination, therefore, GH-activation of Rap $1 / 2$ provides a mechanism for "switching" between ERK1/2 and JNK mediated activities [20].

The most striking evidence for JAK2 independent signalling has come from recent in vivo studies by Barclay et al. [71]. They showed, in mice with a mutated JAK2 proline rich binding motif in Box 1 of the GHR, that Src and ERK1/2 were activated by GH injection but JAK2, STATs 3 and 5 or Akt were not. The mice had a dwarf phenotype, illustrating the key role of JAK2 signalling in growth regulation by $\mathrm{GH}$.

\section{Negative Regulators of GHR Signalling}

The Suppressors of Cytokine Signalling (SOCS) family are negative feedback regulators of cytokine receptor signalling. With respect to modulating GH action and GHR signalling SOCS1, 2, 3 and CIS (cytokine inducible SH2 protein) are the key factors involved [reviewed in 72]. Each of these SOCs is induced by GH and each can inhibit GHR signalling through several mechanisms (Fig. 3):

A. Direct binding to JAK2, thereby blocking JAK2 kinase activation (SOCS 1, 2, 3) [73]

B. Direct binding to phosphorylated tyrosines in the GHR (SOCS 2, 3 and CIS) [74, 75], thereby competitively inhibiting STAT5b binding (SOCS2, CIS). The SOCS3 GHR binding sites are not primary STAT5 sites and so it appears that its actions are through steric inhibiton of JAK phosphorylation [74].

C. Induced proteosomal degradation of GHR through increased ubiquitination due to a role as a ubiquitin ligase (SOCS 1, 2, CIS) [76].

The effects of SOCS however are complex and vary in their relative effectiveness as GHR signalling modulators, both in vitro and in vivo. This is reflected in comprehensive studies with SOCS knockout mouse models. SOCS1 ${ }^{-1-}$ mice are contradictorily growth retarded (implying it is a positive regulator of GH signalling) [77] and the physiological relevance of its in vitro inhibitory effects due to overexpression have therefore been questioned. Neither $\mathrm{SOCS}^{-/-}$nor $\mathrm{CIS}^{-/-}$mice demonstrate any growth phenotype $[72,78]$, therefore raising the issues of functional redundancy across the family. SOCS2 ${ }^{-/}$mice on the other hand are large, signifying that SOCS2 may be the major SOCS involved [79]. STAT5b is required for SOCS2 ${ }^{-/}$gigantism [80]. GH deficiency reverses gigantism and $\mathrm{GH}$ replacement in $\mathrm{GH}$ deficient SOCS2 $^{-/-}$demonstrate increased GH sensitivity [81]. Interestingly, SOCS2 has also been shown to interact with the IGF-I receptor and therefore it may have a major coordinating role in growth regulation.

The Protein Tyrosine Phosphatases (PTPs) are another significant group of negative regulators of $\mathrm{GH} / \mathrm{GHR}$ signalling (Fig. 3D). Three such PTPs have been linked to the GHR - SHP-1 (or PTP-1), PTP-1b and PTP-H1 [reviewed in 72]. SHP-1 is activated by GH and can bind and inhibit STAT5b [82]. Both SHP-1and PTP-1b can interact directly with JAK2 and regulate dephosphorylation of the GH-activated form $[83,84]$. PTP1b can also bind directly to activated GHR and cause dephosphorylation [85]. PTP-H1 also binds directly to and inactivates the GH-induced phosphorylated GHR, thereby inhibiting recruitment of SH2 and other cytoplasmic signalling molecules [85]. Intriguingly, another PTP, SHP-2, has been reported to be both a positive and negative regulator of GHR signalling [86, 87]. The underlying mechanisms for SHP-2 function have not been elucidated.

Signal regulatory protein $\alpha(\operatorname{SIRP} \alpha)$ is a transmembrane glycoprotein which is phosphorylated in a JAK2-dependent manner and can then associate with at least the PTP SHP-2. SIRP $\alpha$ can negatively regulate GH-activated signalling [88] by inhibiting the phosphorylation of JAK2, STAT5b, STAT3 and ERK $1 / 2$, perhaps by binding to SHP-1 or sequestering the GHR positive regulator SHP-2 or JAK2 [86] (Fig. 3E).

\section{Signalling Crosstalk}

Evidence is now emerging of significant cross-talk between the GHR and other receptor signalling systems.

GH-induced phosphorylation of the ErbB family of receptors, including the EGFR, has been shown to regulate EGF responsiveness [reviewed in 89]. Yamauchi et al. [90] have shown that GH, via a JAK2 dependent mechanism, can directly tyrosine phosphorylate the EGFR in vivo and in vitro and that this was independent of the intrinsic tyrosine kinase of the EGFR itself. It led to enhanced binding and activation of Grb2 and subsequent activation of ERK1/2. In adipocytes, GH also led to EGFR serine/threonine phosphorylation, but this was indirect via ERK1/2 [91]. This led to enhanced EGF-stimulated ERK and reduced EGF-induced EGFR down-regulation, both mechanisms that enhance/prolong the effects of EGF.

Both GH and insulin are known key mediators of growth and metabolic regulation. Both hormones utilise common signalling pathways and recent evidence demonstrates that they can directly interact and influence each other's effects by signalling cross-talk. As reviewed recently by $\mathrm{Xu}$ \&Messina [92] insulin regulation of $\mathrm{GH}$ signalling is dependent on the exposure time to insulin - short exposure stimulating the GH-activated MAPK pathway downstream of the GHR itself, longer exposure having the reverse effect and utilising both receptor and downstream mechanisms. In contrast long-term high dose GH has an inhibitory effect on insulin signalling pathways (IRS-PI3K) and may reflect a mechanistic explanation of the induction of insulin resistance.

The relationship between GH and IGF-1 in mechanisms underlying cell proliferation and growth is well known. Recent data however suggests a much closer functional interaction than previously thought. Studies by Frank et al. $[93,94]$ have indicated that GH can induce formation of a functional complex between the GHR, JAK2 and the type 1 insulin-like growth factor receptor (IGF-1R). Deletion of the 


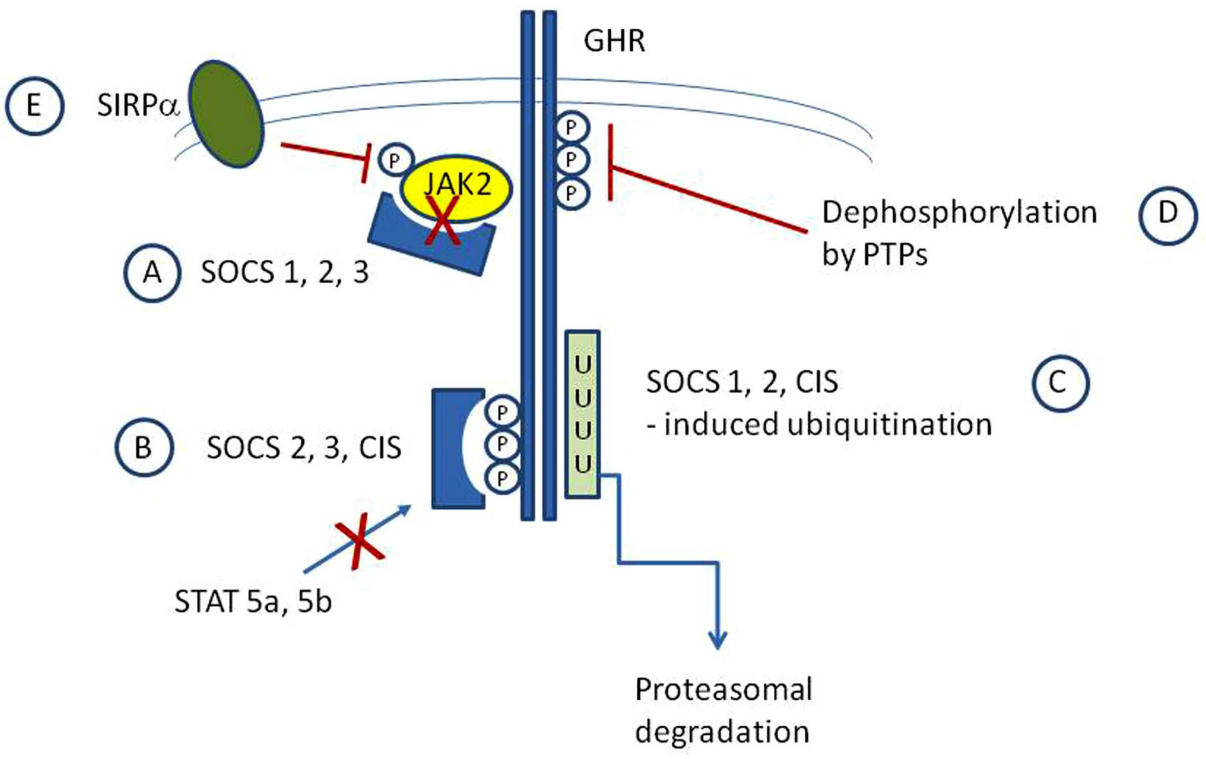

Fig. (3). A proposed model for the actions of the negative regulators of GH-GHR action - Suppressors of Cytokine Signalling (SOCS), Signal Regulatory Protein $\alpha(\operatorname{SIRP} \alpha)$, Protein Tyrosine Phosphatases (PTPs). A) Direct binding to and inhibition of phosphorylation of JAK2 by SOCs 1, 2, 3. B) Direct binding of SOCS 2, 3 or CIS to phosphorylated tyrosines on the GHR, thereby competitively inhibiting binding of STATs. C) SOCs 1, 2 or CIS-induced ubiquitination of the GHR, thereby facilitating proteosomal degradation of the GHR. D) Dephosphorylation by PTPs of phosphorylated GHR. Some PTPs are also able to bind directly to and inhibit STATs and JAK2 (not illustrated). E) SIRP $\alpha$, a transmembrane glycoprotein, inhibits phosphorylation of JAK2 (and STATs - not illustrated), most likely through an interaction with the PTPs.

IGF-1R in primary osteoblasts reduced GH signalling through STAT5 [95]. Additionally, they have demonstrated that the GH/STAT5 dependence on IGF-1R is at least partially independent of the intrinsic tyrosine kinase activity of IGF-1R, or indeed of IGF-1 interaction with the IGF-1R. Nonetheless, they propose that IGF may enhance the effects of $\mathrm{GH}$ through the GHR/JAK2/IGF-1R complex [95]. Intriguingly, however, there was no effect on GH-stimulated ERK signalling, confirming - as discussed above - the capacity for STAT5 independent-JAK2-dependent or independent GH/GHR signalling.

\section{NUCLEAR LOCALISATION AND ACTION OF THE GHR}

While classically regarded as a cell membrane-bound receptor, more than 10 years ago it was reported that both the full-length GHR and its extracellular domain (the GHBP) were also found to be present in the cell nucleus of many cells $[96,97]$. Nuclear localisation occurred in a GH- and STAT5/serum starvation-dependent manner $[96,98]$ and has been associated with rapidly proliferating cells eg liver regeneration, in chronic liver disease and tumour cells [96, 99]. These observations, surprising at the time, do however reflect the localisation and action of other membrane-bound tyrosine kinase receptors (eg EGFR, FGFR, PRLR [reviewed in 99]).

Subsequent studies have extended these observations to provide insight into the mechanisms of nuclear transport and the function of nuclear GHR. The wild-type GHR does not contain a classical nuclear localisation signal (NLS) and it has been suggested that nuclear localisation of either the full length or extra cellular domain depends on association with an NLS-containing carrier protein and a "piggy-back" mechanism involving importin $\beta$ [100]. Engineering an NLS into the GHR increased sensitivity to GH, specifically autocrine GH [100] - which is expressed in a number of tumour cells and is oncogenic when over-expressed [101, 102] - and led to increased and constitutive cellular proliferation [98].

The GHBP, and by implication the nuclear GHR, has been shown to act as a transcription factor - capable of direct transactivation in a mammalian $\beta$-galactosidase reporter assay $[100,103]$. Affinity chromatography of yeast nuclear extracts identified a number of specific GHBP-interacting proteins with mammalian homologues, including a nucleoporin (Nup145) which may be involved in GHR/GHBP nuclear import, the translation regulator EF1a, Coactivator activator (CoAA) and the thyroid receptor (TR)uncoupling protein. The binding to these proteins had an absolute requirement for the second serine in the WSXWSrelated conserved motif [100]. Only the interaction with CoAA has been further examined and validated by GSTpulldown and co-immunoprecipitation assays. Importantly, the association occurs only in the presence of $\mathrm{GH}$. Overexpression of CoAA in GH-responsive cells leads to a specific increased proliferative response to GH. CoAA is a known coactivator of a number of nuclear steroid receptors and has roles in mRNA splicing regulation [104]. CoAA contains several NLS sequences and could therefore also be involved in the "piggyback" nuclear translocation via importin $\beta$. Nuclear expression of GHR in cancer cells shows an association with the frequent over-expression of CoAA [105]. Together these observations suggest a key role for the nuclear GHR-CoAA complex in mediating the cancer- 
related effects of GH. Surprisingly, additional investigations into the transcriptional and other roles of nuclear GHR/GHBP have not been published in the past 2 years. Much work remains to be done to determine the physiological and/or pharmacological implications of this novel localisation and function.

\section{CONCLUSION}

The diverse and central roles of the GH-GHR axis in physiology and pathophysiology have been well recognised for a long time, but the complexities of its varied mechanisms of action are still emerging. This review has provided a broad summary of the GH-GHR signalling pathways as now understood and reflects the increasingly recognised commonalities amongst Class 1 cytokine receptor mechanisms and the cross talk and interaction (direct or indirect) between other receptor activation pathways (eg EGFR and IGF-1R). The identification, but still limited knowledge, of the nuclear localisation and nuclear function of the GHR is a clear example of the many surprises that are predicted to emerge in the continually changing paradigm of GH action.

\section{CONFLICT OF INTEREST}

The authors confirm that this article content has no conflict of interest.

\section{ACKNOWLEDGEMENT}

Declared none.

\section{REFERENCES}

[1] Tsushima T, Friesen HG. Radioreceptor assay for human growth hormone. J Clin Endocrinol Metab 1973; 37: 334-7.

[2] Leung DW, Spencer SA, Cachianes G, et al. Growth hormone receptor and serum binding protein: purification, cloning and expression. Nature 1987; 330: 537-43.

[3] De Vos AM, Ultsch M, Kossiakoff AA. Human growth hormone and extracellular domain of its receptor: crystal structure of the complex. Science 1992; 255: 306-12.

[4] Wells JA. Binding in the growth hormone receptor complex. Proc Natl Acad Sci USA 1996; 93: 1-6.

[5] Argetsinger LS, Campbell GS, Yang X, et al. Identification of JAK2 as a growth hormone receptor-associated tyrosine kinase. Cell 1993; 74: 237-44.

[6] Rowlinson SW, Behncken SN, Rowland JE, et al. Activation of chimeric and full length growth hormone receptors by growth hormone receptor antibodies. A specific conformational change may be required for full-length receptor signalling. J Biol Chem 1998; 273: 5307-14.

[7] Gent J, Van Kerhof P, Roza M, et al. Ligand-independent growth hormone receptor dimerization occurs in the endoplasmic reticulum and is required for ubiquitin system-dependent endocytosis. Proc Natl Acad Sci USA 2002; 99: 9858-63.

[8] Brown RJ, Adams JJ, Pelekanos RA, et al. Model for growth hormone receptor activation based on subunit rotation within a receptor dimer. Proc Natl Acad Sci USA 2005; 12: 814-21.

[9] Kopchick JJ, Parkinson C, Stevens EC, Trainer PJ. Growth hormone receptor antagonists: discovery, development and use in patients with acromegaly. Endocr Rev 2002; 23: 623-46.

[10] Birzniece V, Sata A, Ho KKY. Growth hormone receptor modulators. Rev Endocr Metab Disord 2009; 10: 145-56.

[11] Wilkinson IR, Ferrandis E, Artymiuk PJ, et al. A ligand-receptor fusion of growth hormone forms a dimer and is a potent longacting agonist. Nat Med 2007; 13: 1108-13.

[12] Rowlinson SW, Barnard R, Baastiras S, et al. A growth hormone agonist produced by targeted mutagenesis at binding site 1 .
Evidence that site 1 regulates bioactivity. J Biol Chem 1995; 270: 16833-39.

[13] Wan Y, McDevitt A, Shen B, et al. Increased site 1 affinity improves biopotency of porcine growth hormone. Evidence against diffusion dependent receptor dimerization. J Biol Chem 2004; 279: 44775-84.

[14] Higham CE, Trainer PJ. Growth hormone excess and the development of growth hormone receptor antagonists. Exp Physiol 2010; 93: 1157-69.

[15] Chen WY, Wight DC, Wagner TE, Kopchick JJ. Glycine 119 of bovine growth hormone is critical for growth hormone activity. Mol Endocrinol 1991; 5: 1845-52.

[16] Chen WY, Chen NY, Yun J, et al. In vitro and in vivo studies of antagonistic effects of human growth hormone analogues. J Biol Chem 1994; 269: 15892-97.

[17] Fuh G, Cunningham BC, Fukunaga R, et al. Rational design of potent antagonists to the human growth hormone receptor. Science 1992; 256: 1677-80.

[18] Yang N, Langenheim JF, Wang X, et al. Activation of growth hormone receptors by growth hormone and growth hormone antagonist dimers: insights into receptor triggering. Mol Endocrinol 2008; 22: 978-88.

[19] Waters MJ. The growth hormone receptor. In: Kostyo, Ed. The Handbook of Physiology. Oxford: Oxford University Press 1997; vol. 5: pp. 1301-48.

[20] Shafei F, Herington AC, Lobie PE. Mechanisms of signal transduction utilized by growth hormone. In: Nyberg F, Ed. The somatotrophic axis in brain function. USA: Elsevier Inc 2006; pp. 39-49.

[21] Brooks AJ, Wooh JW, Tunny KA, Waters MJ. Growth hormone receptor; mechanism of action. Int J Biochem Cell Biol 2008; 40: 1984-9.

[22] Frank SJ, Fuchs SY. Modulation of growth hormone receptor abundance and function: roles for the ubiquitin-proteasome system. Biochim Biophys Acta 2008; 1782: 785-94.

[23] He K, Loesch K, Cowan JW, et al. JAK2 enhances the stability of the mature GH receptor. Endocrinology 2005; 145: 4755-65.

[24] Loesch K, Deng L, Wang X, et al. Endoplasmic reticulumassociated degradation of growth hormone receptor in Janus kinase2-deficient cells. Endocrinology 2007; 148: 5955-65.

[25] Loesch K, Deng L, Cowan X, et al. JAK2 influences growth hormone receptor metalloproteolysis. Endocrinology 2006; 147: 2839-49.

[26] Moulin S, Bouzinba-Segard H, Kelly PA, Finidori J. JAK2 and proteasome activities control the availability of cell surface growth hormone receptors during ligand exposure. Cell Signal 2003; 15: 47-55.

[27] Saito Y, Teshima R, Yamazaki $\mathrm{T}$, et al. Ligand-induced internalization and phosphorylation-dependent degradation of growth hormone receptor in human IM-9 cells. Mol Cell Endocrinol 1994; 106: 67-74.

[28] Alves dos Santos CM, ten Broeke T, Strous GJ. Growth hormone receptor ubiquitination, endocytosis and degradation are independent of signal transduction via Janus kinase 2. J Biol Chem 2001; 276: 32635-41.

[29] Deng L, He K, Wang X, et al. Determinants of growth hormone receptor downregulation. Mol Endocrinol 2007; 21: 1537-51.

[30] Wei Y, Rhani Z, Goodyer CG. Characterization of growth hormone receptor messenger ribonucleic acid variants in human adipocytes. J Clin Endocrinol Metab 2006; 91: 1901-8.

[31] Urbanek M, MacLeod JN, Cooke NE, et al. Expression of a human growth hormone (hGH) receptor isoform is predicted by tissuespecific alternative splicing of exon 3 of the hGH receptor gene transcript. Mol Endocrinol 1992; 6: 279-87.

[32] Mercado M, Davila N, McLeod JF, Baumann G. Distribution of growth hormone receptor messenger ribonucleic acid containing and lacking exon 3 in human tissues. J Clin Endocrinol Metab 1994; 78: 731-5.

[33] Dos Santos C, Essioux L, Teinturier C, et al. A common polymorphism of the growth hormone receptor is associated with increased responsiveness to growth hormone. Nat Genet 2004; 36 : $720-4$.

[34] Wassenaar MJ, Dekkers OM, Pereira AM, et al. Impact of the exon 3-deleted growth hormone $(\mathrm{GH})$ receptor polymorphism on baseline height and the growth response to recombinant human GH 
therapy in GH-deficient (GHD) and non-GHD children with short stature: a systematic review and meta-analysis. J Clin Endocrinol Metab 2009; 94: 3721-30.

[35] Bernabeu I, Alvarez-Escolá C, Quinteiro C, et al. The exon 3deleted growth hormone receptor is associated with better response to Pegvisomant therapy in acromegaly. J Clin Endocrinol Metab 2010; 95; 222-9.

[36] Dastot F, Sobrier ML, Duquesnoy P, et al. Alternatively spliced forms in the cytoplasmic domain of the human growth hormone $(\mathrm{GH})$ receptor regulate its ability to generate a soluble $\mathrm{GH}$-binding protein. Proc Natl Acad Sci USA 1996; 93: 10723-8.

[37] Ross RJM, Esposito N, Shen XY, et al. A short isoform of the human growth hormone receptor functions as a dominant negative inhibitor of the full-length receptor and generates large amounts of binding protein. Mol Endocrinol 1997; 11: 265-73.

[38] Ymer SI, Herington AC. Evidence for the specific binding of growth hormone to a receptor-like protein in rabbit serum. Mol Cell Endocrinol 1985; 41: 153-61.

[39] Baumann G, Stolar MW, Amburn K, et al. A specific growth hormone-binding protein in human plasma: initial characterization. J Clin Endocrinol Metab 1986; 62: 134-41.

[40] Herington AC, Ymer SI, Stevenson JL. Identification and characterization of specific binding proteins for $\mathrm{hGH}$ in normal human serum. J Clin Invest 1986; 77: 1817-23.

[41] Baumann G, Amburn KD, Buchanan TA. The effect of circulating growth hormone-binding protein on metabolic clearance, distribution, and degradation of human growth hormone. J Clin Endocrinol Metab 1987; 64: 657-60.

[42] Lim L, Spencer SA, Mckay P, Waters MJ. Regulation of growth hormone $(\mathrm{GH})$ bioactivity by a recombinant human GH-binding protein. Endocrinology 1990; 127: 1287-91.

[43] Herington AC. Growth hormone binding proteins and their relationship to the growth hormone receptor. Endocrinol Metab 1994; 1(Suppl A): 9-15.

[44] Wang X, He K, Gerhart M, et al. Metalloprotease-mediated GH receptor proteolysis and GHBP shedding. Determination of extracellular domain stem region cleavage site. J Biol Chem 2002; 277: 50510-19.

[45] Guan R, Zhang Y, Jiang J, et al. Phorbol ester and growth factorinduced growth hormone $(\mathrm{GH})$ receptor proteolysis and $\mathrm{GH}$ binding protein shedding: relationship to $\mathrm{GH}$ receptor downregulation. Endocrinology 2001; 142: 1137-47.

[46] Wang X, Jiang J, Warram J, et al. Endotoxin-induced proteolytic reduction in hepatic growth hormone receptor: a novel mechanism for GH insensitivity. Mol Endocrinol 2008; 22: 1427-37.

[47] Cowan JW, Wang X, Guan R, et al. Growth hormone receptor is a target for presenilin-dependent gamma-secretase cleavage. J Biol Chem. 2005; 280: 19331-42.

[48] Ihle JN, Witthuhn BA, Quelle FW, et al. Signaling through the hematopoietic cytokine receptors. Ann Rev Immunol 1995; 13: 369-98.

[49] Argetsinger LS, Kouadio JL, Steen H, et al. Autophosphorylation of JAK2 on tyrosines 221 and 570 regulates its activity. Mol Cell Biol 2004; 24: 4955-67.

[50] Smit LS, Meyer DJ, Billestrup N, et al. The role of the growth hormone $(\mathrm{GH})$ receptor and JAK1 and JAK2 kinases in the activation of Stats 1, 3, and 5 by GH. Mol Endocrinol 1996; 10: 519-33.

[51] Johnston JA, Kawamura AM, Kirken RA, et al. Phosphorylation and activation of the Jak-3 Janus kinase in response to interleukin2. Nature 1994; $37: 151-3$.

[52] Hansen LH, Wang X, Kopchick JJ, et al. Identification of tyrosine residues in the intracellular domain of the growth hormone receptor required for transcriptional signalling and Stat5 activation. J Biol Chem1996; 271: 12669-73.

[53] Yang N, Huang Y, Jiang J, et al. Caveolar and lipid raft localisation of the growth hormone receptor and its signalling elements: impact on growth hormone signalling. J Biol Chem 2004; 279: 20898-905.

[54] Herrington J, Smit LS, Schwartz J, Carter-Su C. The role of STAT proteins in growth hormone signaling. Oncogene Res 2000; 19: 2585-97.

[55] Waxman DJ, O'Connor C. Growth hormone regulation of sexdependent liver gene expression. Mol Endocrinol 2006; 20: 261329.
[56] Chia DJ, Ono M, Woelfle J, et al. Characterisation of distinct Stat5b binding sites that mediate growth hormone-stimulated IGF-I gene transcription. J Biol Chem 2006; 281: 3190-7.

[57] Rosenfeld RG, Kofoed E, Buckway C, et al. Identification of the first patient with a conformed mutation of the JAK-STAT system. Pediatr Nephrol 2005; 20: 303-5.

[58] Rojas-Gil AP, Ziros PG, Diaz L, et al. Growth hormone/JAK-Stat axis signal-transduction defect. A novel treatable cause of growth failure. FEBS J 2006; 273: 3453-66.

[59] Zhu T, Goh EL, Graichen R, et al. Signal transduction via the growth hormone receptor. Cell Signal 2001; 13: 599-616.

[60] VanderKuur JA, Butch ER, Waters SB, et al. Signaling molecules involved in coupling growth hormone receptor to MAP kinase activity. Endocrinol 1997; 138: 4301-7.

[61] Piwien-Pilipuk G, Macdougald O, Schwartz J. Dual regulation of phosphorylation and dephosphorylation of C/EBPbeta modulate its transcriptional activation and DNA binding in response to growth hormone. J Biol Chem 2002; 277: 44557-65.

[62] Yamauchi T, Kaburagi Y, Ueki K, et al. Growth hormone and prolactin stimulate tyrosine phosphorylation of insulin receptor substrate-1, -2 , and -3 , their association with p85 phosphatidylinositol 3-kinase (PI3-kinase), and concomitantly PI3kinase activation via JAK2 kinase. J Biol Chem 1998; 273: 1571926.

[63] Zhu T, Goh EL, Lobie PE. Growth hormone stimulates the tyrosine phosphorylation and association of p125 focal adhesion kinase (FAK) with JAK2. FAK is not required for Stat-mediated transcription. J Biol Chem 1998; 273: 10682-9.

[64] Ryu H, Lee JH, Kim KS, et al. Regulation of neutrophil adhesion by pituitary growth hormone accompanies tyrosine phosphorylation of Jak2, p125FAK, and paxillin. J Immunol 2000; 165: 2116-23.

[65] Takahashi MO, Takahashi Y, Iida K, et al. Growth hormone stimulates tyrosine phosphorylation of focal adhesion kinase (p125(FAK)) and actin stress fiber formation in human osteoblastlike cells, Saos2. Biochem Biophys Res Commun 1999; 263: 1006.

[66] Schlaepfer DD, Hunter T. Integrin signaling and tyrosine phosphorylation: just the FAKs? Trends Cell Biol 1998; 8: 151-7.

[67] Chen HC, Guan JL. Stimulation of phosphatidylinositol 3'-kinase association with focal adhesion kinase by platelet-derived growth factor. J Biol Chem 1994; 269: 31223-9.

[68] Ling L, Zhu T, Lobie PE. Src-CrkII-C3G-dependent activation of Rap1 switches growth hormone-stimulated p44/42 MAP kinase and JNK/SAPK activities. J Biol Chem 2003; 278: 27301-11.

[69] Zhu T, Ling L, Lobie PE. Identification of a JAK2-independent pathway regulating growth hormone (GH)-stimulated p44/42 mitogen-activated protein kinase activity. GH activation of Ral and phospholipase D is Src-dependent. J Biol Chem 2002; 277: 45592603.

[70] Rowlinson SW, Yoshizato H, Barclay JL, et al. An agonist-induced conformational change in the growth hormone receptor determines the choice of signalling pathway. Nat Cell Biol 2008; 10: 740-7.

[71] Barclay JL, Kerr LM, Arthur L, et al. In vivo targeting of the growth hormone receptor (GHR) Box1 sequence demonstrates that the GHR does not signal exclusively through JAK2. Mol Endocrinol 2010; 24: 204-17.

[72] Flores-Morales A, Greenhalgh CJ, Norstedt G, Rico-Bautista E. Negative regulation of growth hormone receptor signalling. Mol Endocrinol 2006; 20: 241-53.

[73] Yasukawa H, Misawa H, Sakamoto H, et al. The JAK-binding protein JAB inhibits Janus tyrosine kinase activity through binding in the activation loop. EMBO J 1999; 18: 1309-20.

[74] Hansen JA, Lindberg K, Hilton DJ, et al. Mechanism of inhibition of growth hormone receptor signalling by suppressor of cytokine signalling proteins. Mol Endocrinol 1999; 13: 1832-43.

[75] Ram PA, Waxman DJ. SOCS/CIS protein inhibition of growth hormone-stimulated STAT5 signaling by multiple mechanisms. J Biol Chem 1999; 274: 35553-61.

[76] Kamura T, Maenaka K, Kotoshiba S, et al. VHL-box and SOCSbox domains determine binding specificity for Cul2-Rbx1 and Cul5-Rbx2 modules of ubiquitin ligases. Genes Dev 2004; 18: 3055-65.

[77] Starr R, Metcalf D, Elefanty AG, et al. Liver degeneration and lymphoid deficiencies in mice lacking suppressor of cytokine signaling-1. Proc Natl Acad Sci USA 1998; 95: 14395-99. 
[78] Croker BA, Krebs DL, Zhang JG, et al. SOCS3 negatively regulates IL-6 signalling in vivo. Nat Immunol 2003; 4: 540-45.

[79] Rico-Bautista E, Greenhalgh CJ, Tollet-Egnell P, et al. SOCS2 deficiency induces molecular and metabolic changes that partially overlap with GH-dependent effects. Mol Endocrinol 2005; 19: 78193.

[80] Greenhalgh CJ, Bertolino P, Asa SL, et al. Growth enhancement in suppressor of cytokine signaling 2 (SOCS-2)-deficient mice is dependent on signal transducer and activator of transcription $5 \mathrm{~b}$ (STAT5b). Mol Endocrinol 2002; 16: 1394-406.

[81] Greenhalgh CJ, Rico-Bautista E, Lorentzon M, et al. SOCS2 negatively regulates growth hormone action in vitro and in vivo. $\mathrm{J}$ Clin Invest 2005; 115: 397-406.

[82] Ram PA, Waxman DJ. Interaction of growth hormone-activated STATs with SH2-containing phosphotyrosine phosphatase SHP-1 and nuclear JAK2 tyrosine kinase. J Biol Chem 1997; 272: 17694702.

[83] Hackett RH, Wang YD, Sweitzer S, et al. Mapping of a cytoplasmic domain of the human growth hormone receptor that regulates rates of inactivation of Jak2 and Stat proteins. J Biol Chem 1997; 272: 11128-32

[84] Gu F, Dube N, Kim JW, et al. Protein tyrosine phosphatase 1B attenuates growth hormone-mediated JAK2-STAT signaling. Mol Cell Biol 2003; 23: 3753-62.

[85] Pasquali C, Curchod ML, Walchli S, et al. Identification of protein tyrosine phosphatases with specificity for the ligand-activated growth hormone receptor. Mol Endocrinol 2003; 17: 2228-39.

[86] Kim SO, Jiang J, Yi W, et al. Involvement of the Src homology 2containing tyrosine phosphatase SHP-2 in growth hormone signalling. J Biol Chem 1998; 273: 2344-54.

[87] Stofega MR, Herrington J, Billestrup N, Carter-Su C. Mutation of the SHP-2 binding site in growth hormone $(\mathrm{GH})$ receptor prolongs GH-promoted tyrosyl phosphorylation of GH receptor, JAK2 and STAT5b. Mol Endocrinol 2000; 14: 1338-50.

[88] Stofega MR, Argetsinger LS, Wang H, et al. Negative regulation of growth hormone receptor/JAK2 signaling by signal regulatory protein $\alpha$. J Biol Chem 2000; 275: 28222-9.

[89] Frank SJ. Mechanistic aspects of crosstalk between GH and PRL and Erb receptor family signalling. J Mammary Gland Biol Neoplasia 2008; 13: 119-29.

[90] Yamauchi T, Ueki K, Tobe K, et al. Tyrosine phosphorylation of the EGF receptor by the kinase Jak2 is induced by growth hormone. Nature 1997; 390: 91-6.
[91] Huang Y, Chang Y, Wang X, et al. Growth hormone alters epidermal growth factor receptor binding affinity via activation of ERKs in 3T3-F442A cells. Endocrinology 2004; 145: 3297-306.

[92] Xu J, Messina JL. Crosstalk between growth hormone and insulin signalling. Vitam Horm 2009; 80: 125-53.

[93] Huang Y, Kim SO, Yang N, et al. Physical and functional interaction of growth hormone and IGF-I signalling elements. Mol Endocrinol 2004; 18: 1471-85.

[94] $\mathrm{Xu} \mathrm{J}$, Keeton $\mathrm{AB}$, Franklin JL, et al. Insulin enhances growth hormone induction of the MEK/ERK signalling pathway. J Biol Chem 2006; 281: 982-92.

[95] Gan Y, Zhang Y, Di Girolamo DJ, et al. Deletion of IGF-1 receptor (IGF-1R) in primary osteoblasts reduces GH-induced STAT5 signaling. Mol Endocrinol 2010; 24: 644-56.

[96] Lobie PE, Wood TJ, Chen C-M, et al. Nuclear translocation and anchorage of the GH receptor. J Biol Chem 1994; 269: 31735-46.

[97] Lincoln DT, Sinowatz F, Temmim-Baker L, et al. Growth hormone receptor expression in the nucleus and cytoplasm of normal and neoplastic cells. Histochem Cell Biol 1998; 109: 141-59.

[98] Conway-Campbell BL, Wooh JW, Brooks AJ, et al. Nuclear targeting of the growth hormone receptor results in dysregulation of cell proliferation and tumourigenesis. Proc Natl Acad Sci USA 2007; 104: 13331-6.

[99] Johnson HM, Subramaniam PS, Olsnes S, Jans DA. Trafficking and signalling pathways of nuclear localizing protein ligands and their receptors. Bioessays 2004; 26: 993-1004.

[100] Conway-Campbell BL, Brooks AJ, Robinson PJ, et al. The extracellular domain of the growth hormone receptor interacts with Coactivator Activator to promote cell proliferation. Mol Endocrinol 2008; 22: 2190-202.

[101] Perry JK, Emerald BS, Mertani HC, Lobie PE. The oncogenic potential of growth hormone. Growth Horm IGF Res 2006; 16 277-89.

[102] Waters MJ, Barclay JL. Does growth hormone drive breast and other cancers? Endocrinology 2007; 148: 4533-5.

[103] Graichen R, Sandstedt J, Goh EL, et al. The growth hormone binding protein is a location-dependent cytokine receptor transcriptional enhancer. J Biol Chem 2003; 278: 6346-54.

[104] Auboeuf D, Dowhan DH, Li X, et al. CoAA, a nuclear receptor coactivator protein at the interface of transcriptional coactivation and RNA splicing. Mol Cell Biol 2004; 24: 442-53.

[105] Sui Y, Yang Z, Xiong S, et al. Gene amplification and associated loss of 5 ' regulatory sequences of CoAA in human cancers. Oncogene 2007; 26: 822-35. 